\title{
Enjeux et perspectives de l'irrigation des fruits et légumes
}

\author{
Henri Bois \\ Président de la Confédération fruitière, légumière et horticole \\ Daniel Veschambre \\ Adjoint du Chef du Département Environnement et Qualité du Ctifl
}

Les cultures fruitières et légumières irriguées occupent 260000 ha environ soit $22,5 \%$ des surfaces irriguées toutes cultures et $45 \%$ des surfaces consacrées aux fruits et légumes; dans la moitié sud de la France, en dehors de quelques espèces de fruits secs, ou de cas de production de légumes "à sec ", la quasi-totalité de ces cultures est irriguée. Selon l'année, la consommation nette en eau d'irrigation varie de 500 à 750 millions de $\mathrm{m}^{3}$, soit $25 \%$ de la consommation nationale d'eau d'irrigation ; c'est dire l'importance de l'irrigation pour cette filière.

En raison de ces dernières années de sécheresse, la tendance est à l'accroissement des surfaces équipées et des consommations, en particulier pour les productions légumières dans les régions atlantiques (Val de Loire, Bretagne). Mais ceci n'a fait qu'accélérer une tendance liée à des enjeux économiques.

Les exigences commerciales supposent en effet, l'approvisionnement le plus régulier possible en fruits et légumes dans la seule catégorie la plus demandée par le négoce. Ceci revient à éliminer une des causes majeures de fluctuation des quantités et qualités des fruits et légumes: le déficit hydrique. Dans bien des cas, en particulier pour les légumes (serres, région méditerranéenne), l'irrigation est la seule origine d'eau pour l'alimentation des cultures.

La production est donc confrontée à la nécessité de disposer d'eau en quantité variable selon l'espèce et surtout la durée de culture : 500 à $1000 \mathrm{~m}^{3} /$ ha pour des cultures courtes de légumes en plein champ à parfois $4000 \mathrm{ou}$ $5000 \mathrm{~m}^{3} /$ ha pour des arbres fruitiers en région méditerranéenne. Les cultures sous serre peuvent nécessiter plus de $10000 \mathrm{~m}^{3} /$ ha.

\section{Les besoins en irrigation pour les fruits et légumes}

\section{a) Besoins globaux}

Compte tenu des surfaces équipées, les besoins en irrigation selon les espèces sont détaillés dans le tableau 1 pour les productions fruitières pour un total variant de 300 à 450 millions de $\mathrm{m}^{3}$ par an. Les besoins des cultures légumières détaillées dans le tableau 2 , oscillent entre 200, et 300 millions de $\mathrm{m}^{3}$. Dans ces dernières les cultures sous serre et abri ont des besoins stables de l'ordre de 20 millions de $\mathrm{m}^{3}$ par an (tabl. 3).

On peut remarquer également que les surfaces irriguées de quelques productions ont tendance à croître : l'abricot, le melon, la carotte, le haricot et le chou fleur. Ces accroissements se font surtout dans les régions méridionales mais aussi en Bretagne pourle chou fleur et le haricot.

\section{b) Besoins selon les régions et la saison}

La variabilité selon les régions est illustrée par la carte (fig. 1) de fréquence quinquénale pour les arbres fruitiers à pépin.

Les tableaux 4 et 5 donnent une évaluation des besoins pour pommier et pêcher d'une part et pour concombre et tomate hors-sol sous serre d'autre part.

On peut noter en outre deux points importants :

- pour les arbres fruitiers, on peut considérer que les besoins ne sont indispensables que s'ils dépassent $1000 \mathrm{~m}^{3} /$ ha. Or, selon la région c'est le cas 1 année sur 2 à Bergerac, 7 années sur 10 à Toulouse ou Angers, tous les ans à Nîmes ;

- pour les productions sous serre, où la totalité des besoins en eau est assurée par l'irrigation, les pertes sont importantes par drainage dans le cas des cultures hors-sol. Sur les 9000 à $11000 \mathrm{~m}^{3} /$ ha nécessaire, il y a perte de 1800 à $2000 \mathrm{~m}^{3} /$ ha.

Dans tous les cas il s'agit de besoins correspondant à des consommations nettes d'eau. 


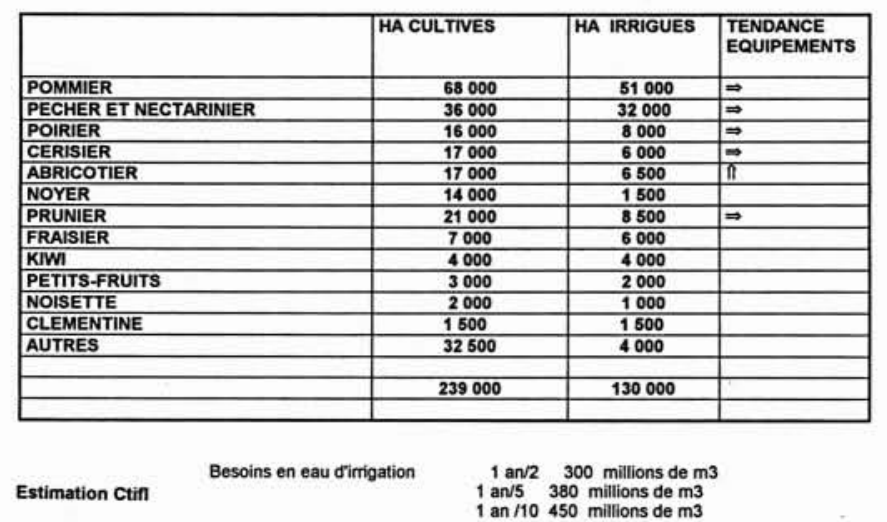

Tableau 2. Surfaces légumières irriguées (serres et plein champ)

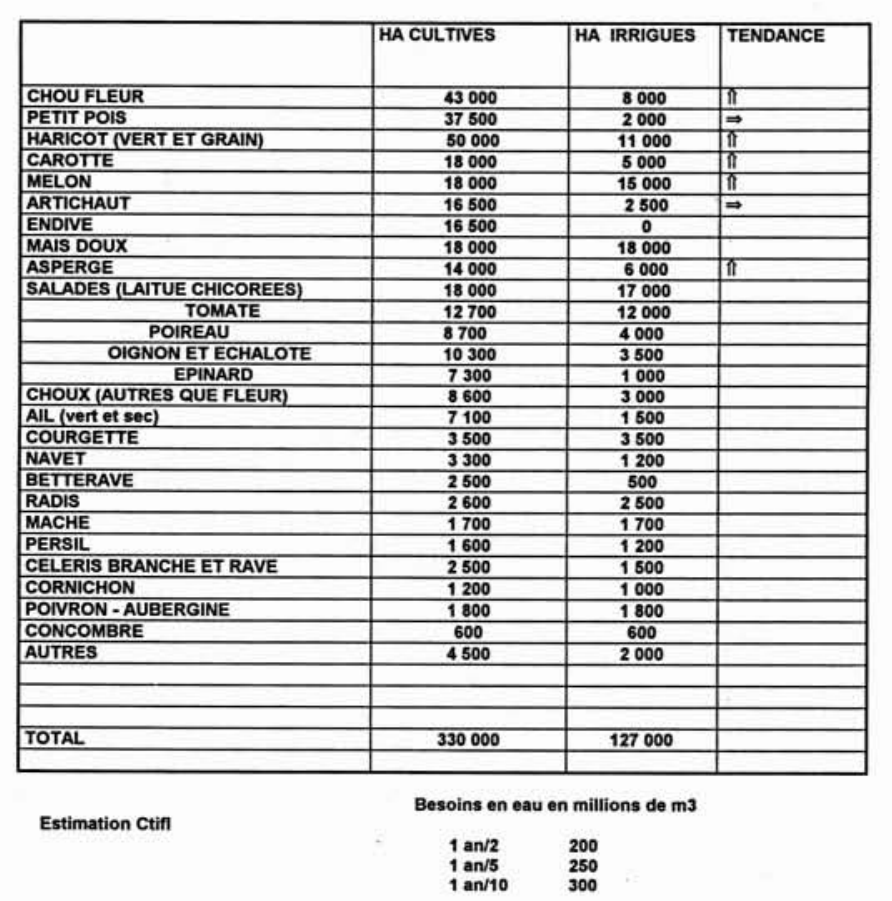

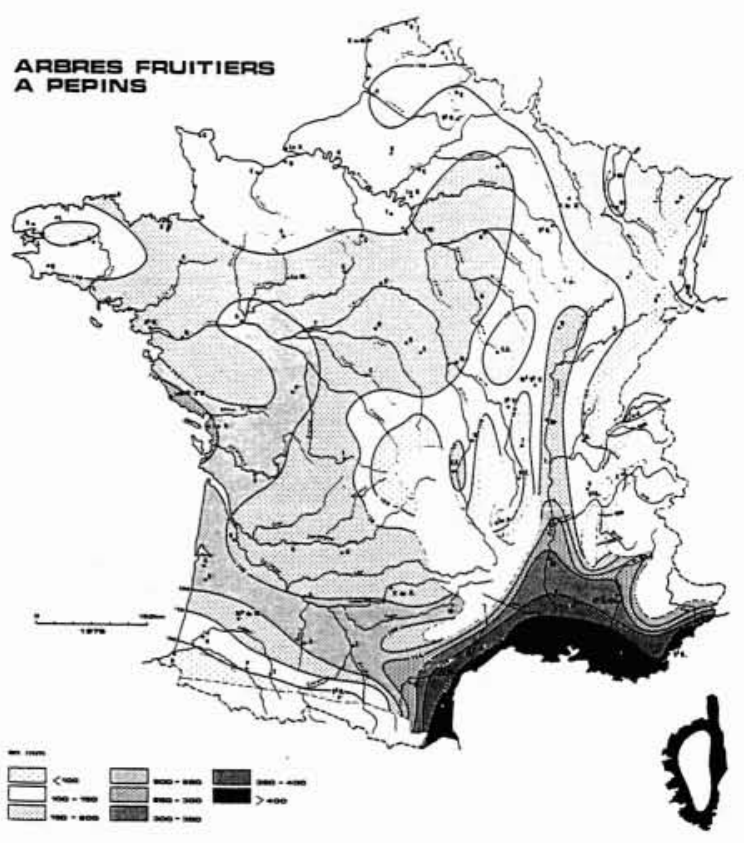

1. Besoins de fréquence quinquennale.

\begin{tabular}{|lr|c|}
\hline \multicolumn{2}{|c|}{$\begin{array}{c}\text { Tableau 3 Estimation des besoins en eau } \\
\text { des cultures sous serre et abris }\end{array}$} \\
\hline \multicolumn{2}{|c|}{ Surface ha } & Million de $\mathrm{m}^{3}$ \\
\hline Chautfees & 1100 & 11 \\
antigel & 600 & 4 \\
froides & 3100 & 15 \\
\cline { 2 - 3 } & 4800 & $\frac{1}{30}$ \\
\hline
\end{tabular}

\begin{tabular}{|l|c|c|}
\hline \multicolumn{3}{|c|}{ Tableau 6. Calibres selón les espèces } \\
\hline Espèco & $\begin{array}{c}\text { Calibre minimal } \\
\text { exigé par la réglementation }\end{array}$ & Callibres rémunérateurs \\
\hline Pormme & $65 \mathrm{~mm}$ & 70 a $85 \mathrm{~mm}$ \\
Péche & $51 \mathrm{~mm}$ & $67 \mathrm{à} 80 \mathrm{~mm}$ \\
Tornate & $47 \mathrm{~mm}$ & $57 \mathrm{a} 77 \mathrm{~mm}$ \\
\hline
\end{tabular}

Tableau 4. Etude fréquentielle des besoins d'irrigation

(besoins exprimés en mm) (Lecarpentier, Scherer, Darves-Bornoz, 1979)

\begin{tabular}{|c|c|c|c|c|c|c|c|c|c|c|}
\hline & ESPECE & FREQUENCE & MAI & JUIN & JULLLLET & AOUT & SEPTEMBRE & $\begin{array}{l}\text { BESOINS } \\
\text { MENSUELS } \\
\text { DE POINTE }\end{array}$ & \begin{tabular}{|l|} 
BESOIN \\
IRRIGATION
\end{tabular} & $\begin{array}{l}\text { \% des ANNEES OU } \\
\text { LES BESONS } \\
\text { IRRIGATION }>100 \mathrm{~mm}\end{array}$ \\
\hline BERGERAC & 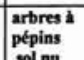 & $\begin{array}{l}1 \mathrm{an} / 2 \\
1 \mathrm{an} / \mathrm{s} \\
1 \mathrm{man} / 0\end{array}$ & $\begin{array}{l}0 \\
0 \\
0\end{array}$ & $\begin{array}{l}0 \\
0 \\
7\end{array}$ & $\begin{array}{l}44 \\
93 \\
118\end{array}$ & $\begin{array}{l}40 \\
88 \\
104\end{array}$ & $\begin{array}{l}0 \\
33 \\
43\end{array}$ & $\begin{array}{l}82 \\
119 \\
133\end{array}$ & $\begin{array}{l}104 \\
188 \\
221\end{array}$ & $53 \%$ \\
\hline ANGERS & $\begin{array}{l}\text { arbreat } \\
\text { peppins } \\
\text { sol nu }\end{array}$ & $\begin{array}{l}1 \mathrm{an} / 2 \\
1 \mathrm{an} / 5 \\
1 \mathrm{an} / 10\end{array}$ & $\begin{array}{l}0 \\
0 \\
0\end{array}$ & $\begin{array}{l}10 \\
22 \\
46\end{array}$ & \begin{tabular}{|l|}
55 \\
98 \\
113
\end{tabular} & \begin{tabular}{|l|}
69 \\
105 \\
115
\end{tabular} & $\begin{array}{l}17 \\
38 \\
49\end{array}$ & $\begin{array}{l}\begin{array}{l}98 \\
124 \\
132\end{array} \\
\end{array}$ & $\begin{array}{l}132 \\
219 \\
257\end{array}$ & $70 \%$ \\
\hline NIMES & 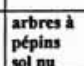 & $\begin{array}{l}1 \mathrm{an} / 2 \\
1 \\
1 \mathrm{an} / 5 \\
1 \mathrm{an} / 10\end{array}$ & $\begin{array}{l}0 \\
1 \\
18\end{array}$ & $\begin{array}{l}40 \\
85 \\
108\end{array}$ & $\begin{array}{l}141 \\
173 \\
183\end{array}$ & \begin{tabular}{|l}
120 \\
152 \\
158
\end{tabular} & $\begin{array}{l}32 \\
57 \\
63\end{array}$ & $\begin{array}{l}159 \\
179 \\
185\end{array}$ & $\begin{array}{l}327 \\
401 \\
438\end{array}$ & $100 \%$ \\
\hline NIMES & $\begin{array}{l}\text { por nu } \\
\text { pecher } \\
\text { sol nu }\end{array}$ & $\begin{array}{l}1 \mathrm{an} / 2 \\
1 \mathrm{an} / \mathrm{s} \\
1 \mathrm{an} / 10\end{array}$ & $\begin{array}{l}0 \\
28 \\
28 \\
81\end{array}$ & \begin{tabular}{|l}
86 \\
130 \\
151
\end{tabular} & \begin{tabular}{|l|l|}
140 \\
169 \\
176
\end{tabular} & \begin{tabular}{|l|}
71 \\
101 \\
105
\end{tabular} & \begin{tabular}{|l}
30 \\
49 \\
49 \\
59
\end{tabular} & \begin{tabular}{|l|l|}
148 \\
1172 \\
181
\end{tabular} & \begin{tabular}{|l|}
301 \\
335 \\
392
\end{tabular} & $100 \%$ \\
\hline TOULOUSE & \begin{tabular}{|l|} 
arbrestì \\
peppins \\
sol nu \\
pecther \\
sol nu
\end{tabular} & $\begin{array}{l}1 \mathrm{an} / 2 \\
11 \mathrm{an} 5 \\
1 \mathrm{an} / 10 \\
1 \mathrm{an} / 2 \\
1 \mathrm{an} / 2 \\
1 \mathrm{ann} / 10\end{array}$ & $\begin{array}{l}0 \\
0 \\
0 \\
0 \\
0 \\
3\end{array}$ & $\begin{array}{l}0 \\
6 \\
37 \\
9 \\
50 \\
82\end{array}$ & \begin{tabular}{|l|}
59 \\
107 \\
123 \\
78 \\
114 \\
127
\end{tabular} & $\begin{array}{l}80 \\
107 \\
117 \\
41 \\
66 \\
75\end{array}$ & $\begin{array}{l}20 \\
38 \\
50 \\
10 \\
34 \\
45\end{array}$ & \begin{tabular}{|l}
102 \\
128 \\
137 \\
94 \\
123 \\
131 \\
131
\end{tabular} & $\begin{array}{l}1773 \\
231 \\
252 \\
143 \\
200 \\
232\end{array}$ & ${ }_{63 \%}^{73 \%}$ \\
\hline
\end{tabular}

Tableau 5. Evaluation des besoins d'irrigation sous serre en culture hors-sol en solution perdue (20 à $25 \%$ de drainage) avec plantation en janvier

\begin{tabular}{|l|l|l|l|l|l|l|l|l|l|l|l|l|l|}
\hline LEU & ESPECES & JANVIER & FEVR & MARS & AVRIL & MAI & JUIN & JUILL & AOUT & SEPT. & OCT. & $\begin{array}{l}\text { BESOINS } \\
\text { TOTAUX mm }\end{array}$ & $\begin{array}{l}\text { BESOINS } \\
\text { JOURNALIERS mm }\end{array}$ \\
\hline NANTES & concombre & 20 & 40 & 70 & 90 & 120 & 90 & 140 & 140 & 120 & 90 & 920 & \\
\hline & tomate & 25 & 45 & 75 & 100 & 125 & 100 & 160 & 160 & 140 & 100 & 1030 & 7 \\
\hline & & & & & & & & & & & & & \\
\hline NIMES & tomate & 30 & 50 & 85 & 120 & 140 & 120 & 185 & 175 & 160 & 120 & 1185 & 8 \\
\hline
\end{tabular}




\section{Les enjeux}

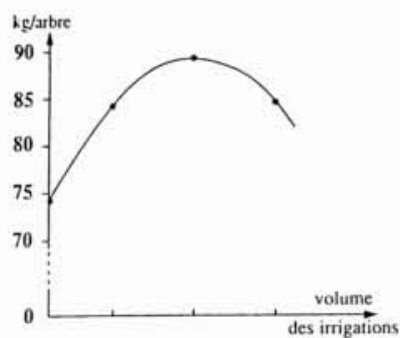

2. Evolution de la production en fonction du volume des irrigations (d'après Colorio G., Strabbioli G., Spada G., 1982).

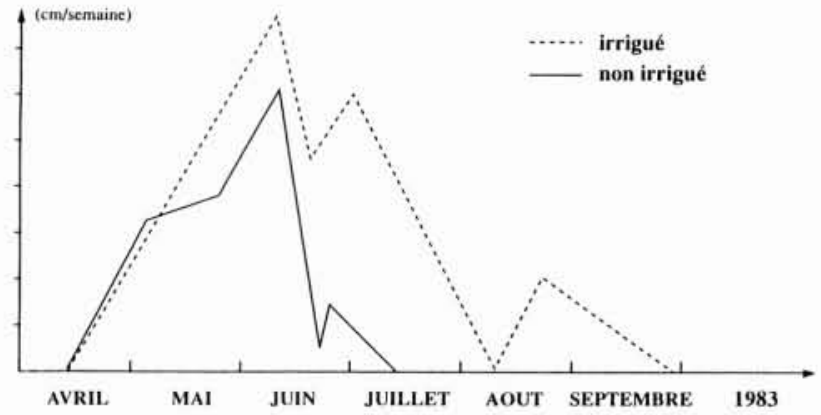

3. Incidence du régime hydrique sur la vitesse de croissance de pousses de cerisiers (Burlat/SL 64 - 3 ans) (d'après P. Soing, J.F. Mandrin, 1986).

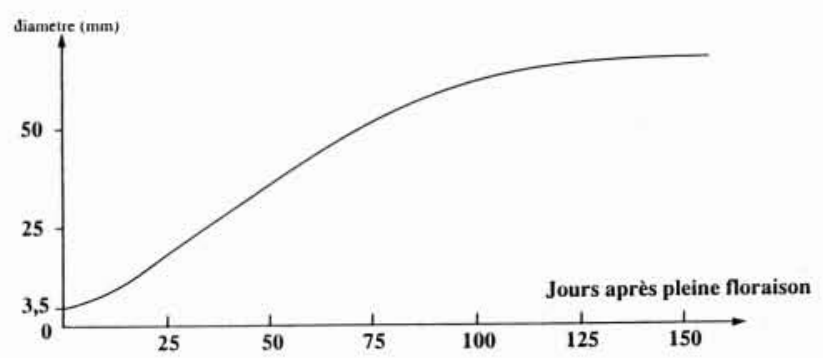

4. Courbes d'évolution du diamètre des fruits. Pomme Golden Delicious en 1982 (d'après H. Magein, 1988).

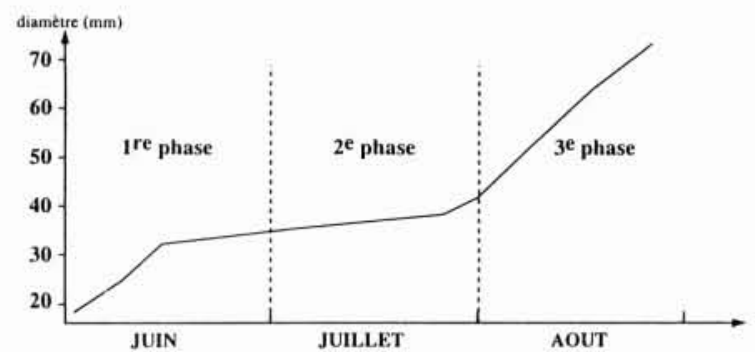

5. Courbes d'évolution du diamètre des fruits. Pêche Merril (d'après S.H. Li, 1989).

\section{a) Enjeux économiques}

- Irrigation et productivité : L'irrigation est un facteur de productivité bien connu (fig. 2). C'est même une condition sine qua non de production dans plusieurs cas:

- cultures sous abri ;

- cultures de plein air de la plupart des légumes et de certains fruits (pomme, poire, pêche) dans les régions méditerranéennes.

Dans le cas des cultures fruitières, il est de plus en plus nécessaire de régulariser la production d'une année sur l'autre. L'irrigation est un des moyens permettant de limiter l'alternance. Elle permet un allongement normal des rameaux et leur mise à fruits (fig. 3).

- Qualité commerciale: Le négoce réclame sans cesse des fruits et légumes dans le calibre le plus marchand qui devient bien souvent le seul vendable et donc rentable.

Or l'irrigation est un des facteurs essentiel permettant de maitriser le calibre.

Un déficit hydrique peut se traduire par des pertes dommageables de calibre. Dans certains cas, l'absence de l'irrigation sur abres fruitiers peut aboutir à une production invendable.

Il faut préciser d'autre part, que l'influence de l'irrigation sur le calibre est liée à l'espèce : arbre à pépins d'une part, arbre à noyau d'autre part. Dans le cas du pommier (fig. 4), l'irrigation est indispensable en phase de multiplication cellulaire. Un déficit à cette période n'est pas rattrapable. Dans le cas des arbres à noyau, figure 5, c'est au cours de la phase finale, que le déficit hydrique est dommageable.

La notion d'extensification devient dans ces conditions difficile à adapter aux productions fruitières et légumières, si elle suppose l'absence d'irrigation. Sans parler des cas où l'irrigation est une condition de production sine qua non, la suppression de l'irrigation dans les vergers existant aurait pour conséquences l'abandon de la culture.

On peut en revanche s'interroger sur une autre conception du verger qui ne serait pas irrigué : faible peuplement, porte-greffe adapté, conduite limitant la charge des arbres de façon à conserver le calibre.

Mais même si un tel concept qui reste à vérifier par l'expérimentation peut techniquement aboutir, qu'en serait-il de la rentabilité ?

Toutefois, dans de nombreux cas, même en Provence et Languedoc, il doit être possible de conduire correctement un verger intensif avec de faibles besoins en eau d'irrigation. Il s'agit de sol où la réserve en eau est importante.

- Coût de l'eau: Le coût de l'irrigation peut être très variable selon les consommations et l'origine de l'eau. On recense une grande variété d'origine d'eau : si des surfaces importantes dans les régions méridionales bénéficient de l'eau distribuée par les Compagnies d'aménagement, il reste de nombreux cas où l'irrigant doit assurer lui-même 
l'approvisionnement : forage, associé ou non à un bassin de stockage, retenue collinaire. Enfin dans des cas limités, les producteurs en particulier de légumes, n'ont pour ressources que le réseau d'eau potable au coût souvent prohibitif $\left(15\right.$ à $\left.20 \mathrm{~F} / \mathrm{m}^{3}\right)$.

\section{b) Enjeux environnementaux}

Ils ont tendance à prendre une part croissante pour les productions de fruits et légumes.

- La disponibilité en eau est loin d'être totale partout où les producteurs en ont besoin. Certes les aménagements collectifs assurent les besoins dans bien des régions. Toutefois, la fourniture ne correspond pas exactement aux besoins: le rythme des "tours d'eau " délivrant de l'eau tous les 3 jours ne permet pas l'irrigation localisée qui suppose au moins une sinon deux ou trois irrigations par jour.

Ailleurs, en l'absence de réseau collectif, le producteur peut se trouver en situation d'absence d'eau dans le proche sous-sol (Périgord - Bretagne - Charentes). Quand aux retenues collinaires, quand elles sont possibles, les caprices météorologiques rendent l'irrigation aléatoire : ces dernières années de sécheresse furent cruelles pour bien des producteurs.

Les tentatives de forages toujours plus profonds pour compenser ces déficits, et aussi pour avoir une eau «propre " sont souvent déçues pour les débits et même pour la qualité : les éventuelles teneurs en fer excessives sont une déconvenue pour l'irrigation goutte à goutte, en raison des risques de bouchage. Enfin, ces pompages dans les nappes profondes comme ceux faits dans les cours d'eau voire les canaux navigables pose le problème général de l'utilisation d'une eau qui n'est pas toujours renouvelable. Mais ceci n'a rien de spécifique aux fruits et légumes.

- Le problème de la disponibilité de l'eau conduit à examiner le problème de la déperdition de l'eau par l'irrigant. Certes, il ne s'agit pas de gaspillage volontaire. Mais les conditions de la conduite de l'irrigation amène à des surirrigations et à une perte d'eau non utilisée, le rendement hydraulique et l'efficience agronomique n'étant jamais de $100 \%$.

Le système d'irrigation à la raie est développé sur plus de 30000 ha principalement sur pommier, pêcher et productions légumières dans les régions méditerranéennes. Ce système conduit à une perte d'eau en profondeur allant de 30 à $40 \%$ de l'eau apportée. Une amélioration considérable a été apportée par le procédé Bas-Rhône qui régularise l'apport sur la ligne et limite les pertes à $20 \%$ environ.

Le système d'apport par aspersion et irrigation localisée (minidiffuseurs, goutte à goutte) sont en théorie peu différents en ce qui concerne le rendement hydraulique. Ceci suppose que la conduite soit parfaitement maîtrisée, en ce qui concerne l'adéquation des apports et des besoins. C'est évidemment loin d'être le cas. Certes les installations mobiles ont été largement remplacées soit par des équipements sous frondaison en couverture totale soit par des réseaux d'irrigation localisée, le plus souvent commandés par programmation (50 000 ha environ). Ceci permet de mieux adapter les apports en fonction de la demande climatique. Toutefois, ceci suppose :

- une bonne maîtrise de la dose et de la fréquence d'apport en fonction du sol et de la culture;

- la tenue d'un bilan hydrique;

- un contrôle régulier de l'humidité du sol.

Largement développées chez les producteurs de fruits et légumes, ces pratiques sont loin d'être généralisées.

Les équipements de programmation qui éliminent bien des soucis au producteur ne favorisent pas le suivi et le contrôle de l'état du sol de chaque parcelle. Si le sol est suffisamment filtrant, il n'y aura pas de sanction technique à la surirrigation (asphyxie racinaire, développement de certaines maladies).

Les pertes en profondeur sont source de pollution. Il s'agit essentiellement des nitrates. Les autres fertilisants sont soit trop peu mobiles (phosphates) soit absorbés par les argiles à plus ou moins grande profondeur avant de parvenir à la nappe.

Il n'est pas possible de donner une quelconque estimation des pertes de nitrates pour les fruits et légumes: la diversité des espèces, des besoins en éléments nutritifs et des pratiques de la fertilisation rend l'approche difficile. Elle l'est d'autant plus que l'on ne dispose pas encore d'outils permettant de bâtir un bilan azotée pour toutes les espèces. En revanche, les méthodes de diagnostic in situ de la teneur en nitrates d'un sol commencent à se développer en particulier pour les cultures légumières.

Quant aux cultures hors sol souvent citées comme génératrices de pollution, on estime que l'effluent constitué par le drainage obligatoire dans le système dit "en solution perdue ", contient en moyenne $2 \mathrm{~g}$ par litre de sels, essentiellement des nitrates, en plus de ce que contient l'eau d'irrigation de départ. Le rejet s'élève, pour un drainage moyen de $2000 \mathrm{~m}^{3} / \mathrm{ha} / \mathrm{an}$ à près de 4 tonnes de $\mathrm{sel} / \mathrm{ha}$. Heureusement ces cultures ne sont pas concentrées en France, comme elles sont en Hollande dans le Westland ou en Espagne à Almeria. La dissémination entre Vaucluse, Bouches-du-Rhône, Pyrénées-Orientales, Lot-et-Garonne, Loire-Atlantique, Finistère, Loiret, ... dilue, si on peut dire, les risques. Surtout la perspective très prochaine de mise au point de la conduite dite en " recyclage des solutions ", doit permettre d'éliminer cette pollution.

\section{Les perspectives}

Les perspectives s'inscrivent dans trois domaines d'actions complémentaires et indissociables.

\section{a) La recherche appliquée et l'expérimentation}

Les programmes déjà démarrés tant en France notamment par le Ctifl, qu'à l'étranger, doivent être intensifiés. Ils concernent : 


\section{IRRIGATION DES FRUITS ET LÉGUMES}

- la maitrise de la conduite de l'irrigation par la mise en œuvre de méthodes de raisonnement du type «production intégrée " et d'automates de pilotage. Ceux-ci, outre les données météorologiques, peuvent intégrer des données liées au sol (humidité au sol : (Humicro 2000) ou à la plante par des mesures micromorphométriques (Pépista)) ;

- la maîtrise de la fertilisation en mettant au point : le calendrier des besoins azotés, des outils de diagnostics aussi bien au niveau du sol que de la plante.

Le nombre d'espèces cultivées rend la tâche longue. Toutefois, le choix de plantes modèles doit permettre de progresser rapidement. Déjà des données ont pu être établies pour quelques espèces ligneuses (pommier, pêcher) et pour quelques plantes à cycles plus court (fraisier, asperge, laitue);

- la maîtrise du recyclage des solutions nutritives en culture hors-sol, essentiellement pour la tomate et le concombre.

\section{b) Les actions de développement}

Il est nécessaire de renforcer ces actions réalisées par tous les acteurs (Chambre d'Agriculture, Groupements techniques, Compagnies d'aménagement) de façon, non seulement à faire connaître les règles et techniques de conduite de l'irrigation et de la fertilisation, mais surtout à faire prendre conscience des enjeux. Ce n'est qu'à cette condition que des efforts financiers pourront être consentis.

\section{c) Les investissements}

Ils consistent principalement à :

- constituer des réserves d'eau à tous les niveaux pour éviter toute rupture d'approvisionnement. Ces réserves peuvent être collectives ou individuelles. Mais elles s'avèrent indispensables. Les risques de pluviométrie nulles ou quasiment pendant les mois d'irrigation, conduit à des prévisions de stockage équivalente aux besoins en irrigation d'une saison.

Sans ces réserves, il sera illusoire de vouloir produire sans risque des fruits et légumes de qualité commerciale requise dans bien des régions de production. Cette nécessité se trouve renforcée par le risque de baisse de niveau des fleuves et rivières lors d'année de sécheresse, lorsqu'elles sont les seules origines d'eau d'irrigation ;

- acquérir et maîtriser les outils permettant d'ajuster au mieux les apports des données météorologiques permet une amélioration considérable du rendement hydraulique. $\mathrm{Ce}$ type d'équipement est encore trop peu répandu.

Mais à l'heure où l'eau est de plus en plus nécessaire, rare et chère, ces investissements permettront la poursuite d'une production fruitière et légumière performante. 